\title{
Caracterização clínica e sociodemográfica de pessoas estomizadas atendidas em um centro de referência
}

\author{
Clinical and sociodemographic characterization of ostomized patients treated \\ at a referral center
}

\author{
Como citar este artigo:
}

Cerqueira LCN, Cacholi SAB, Nascimento VS, Koeppe GBO, Torres VCP, Oliveira PP. Clinical and sociodemographic characterization of ostomized patients treated at a referral center. Rev Rene. 2020;21:e42145. DOI: https://doi.org/10.15253/2175-6783.20202142145
${ }^{1}$ Universidade Veiga de Almeida - Campus Cabo Frio. Cabo Frio, RJ, Brasil.

${ }^{2}$ Polo de Atendimento à Pessoa Estomizada de Cabo Frio. Cabo Frio, RJ, Brasil.

\section{Autor correspondente:}

Luciana da Costa Nogueira Cerqueira Rua Vitor Igrejas, 24, casa 20. Ville Blanche, Cabo Frio. CEP: 28915-210. Rio de Janeiro, RJ, Brasil.

E-mail: luciana.nogueira7@hotmail.com

\begin{abstract}
RESUMO
Objetivo: caracterizar o perfil socioclínico epidemiológico da população com estomia atendida em um centro de referência. Métodos: estudo transversal com 255 prontuários do Serviço de Atenção Básica à Pessoa Ostomizada submetidos a uma análise descritiva e estatística (teste qui-quadrado e teste exato de Fisher). Resultados: 54,1\% eram homens, 56,5\% idosos, 50,2\% brancos, $46,7 \%$ tinham companheiro, 59,2\% com até o ensino fundamental e 34,5\% aposentados. Pessoas com colostomia, 71,4\%, sendo 33,7\% temporárias, $69,4 \%$ por neoplasias e 17,3\% apresentaram dermatite. Somente uma correlação entre presença de complicações e sexo dos pacientes foi observada $(p=0,037)$. Conclusão: os dados indicam predominância de idosos, do sexo masculino, brancos, com companheiro, aposentados e de baixa escolaridade. Pessoas com colostomia temporárias por neoplasia, com dermatite como principal complicação prevaleceram, tendo uma correlação entre o sexo e a complicação apresentada.

Descritores: Estomia; Perfil de Saúde; Cuidados de Enfermagem.
\end{abstract}

\begin{abstract}
Objective: to characterize the epidemiological socio-clinical profile of the population with ostomy treated at a referral center. Methods: cross-sectional study with 255 records of the Primary Care Service for the Ostomized Person submitted to a descriptive and statistical analysis (chi-square test and Fisher's exact test). Results: $54.1 \%$ were men, 56.5\% elderly, $50.2 \%$ white, $46.7 \%$ had a partner, $59.2 \%$ had elementary school and $34.5 \%$ retired. Patients with colostomy, $71.4 \%$, being $33.7 \%$ temporary, $69.4 \%$ due to neoplasms and $17.3 \%$ presented dermatitis. Only a correlation between presence of complications and gender of patients was observed ( $p=0.037)$. Conclusion: the data indicate a predominance of elderly, male, white, with partner, retirees and with a low education level. People with temporary colostomy, due to neoplasia, with dermatitis as the main complication, prevailed, showing a correlation between sex and the presented complication.

Descriptors: Ostomy; Health Profile; Nursing Care.
\end{abstract}




\section{Introdução}

Ao longo dos anos, houve uma mudança sociodemográfica que impactou no processo saúde doença, como urbanização, mudança no hábito, globalização do conhecimento e avanços tecnológicos, o que levou a um aumento da expectativa de vida e, em paralelo, contribuiu para a elevação do número de doenças crônicas não transmissíveis, ascendendo o número de pessoas com estomia. De acordo com a Ostomy Associations of America, estima-se que 150.00 americanos possuem uma estomia e que 130.000 novas cirurgias são efetuadas anualmente. No Brasil, a cada ano aproximadamente um milhão e 400 mil procedimentos cirúrgicos são realizados para confecção de uma estomia $^{(1-3)}$. Os dados epidemiológicos referentes a pessoa com estomia no país ainda são escassos. Neste contexto, o que existe são estudos locais isolados de caracterização dessa população ${ }^{(4)}$.

Os pacientes estomizados estão sujeitos a diferentes alterações biopsicosócioespirituais, por este motivo, o enfermeiro possui papel fundamental na minimização destes impactos. Como atribuições, ele deve desenvolver um planejamento e uma assistência adequada, que inclua um aporte técnico, apoio psicológico e um planejamento de educação em saúde que prepare o paciente para conviver com a estomia, estimular a autonomia e o autocuidado com o intuito de proporcionar uma adaptação do indivíduo a sua nova condição e evitar o surgimento de complicações ${ }^{(2)}$.

A carência de dados epidemiológicos em âmbito nacional e estadual pode interferir diretamente nas implementações de medidas que visem prestar uma assistência direcionada e de qualidade a pessoa estomizada. Sendo assim, este estudo torna-se relevante, pois a caraterização dessa clientela assistida em um serviço especializado auxilia na compreensão do estado situacional de saúde, bem como subsidia dados para implementação de políticas de saúde e direcionamento do planejamento de enfermagem que vise a melhoria da qualidade de assistência dos pacientes. Também fornece dados atualizados e reais aos ges- tores de saúde para o enfrentamento dos problemas relacionados a essa população. Por tanto, objetivou-se caracterizar o perfil socioclínico epidemiológico da população com estomia atendida em um centro de referência.

\section{Métodos}

Trata-se de um estudo descritivo e transversal com uma amostra composta por 255 prontuários de pacientes estomizados. A coleta de dados ocorreu no Polo da Baixada Litorânea do Serviço de Atenção Básica à Pessoa Ostomizada (Polo I), localizado no município de Cabo Frio-RJ, Brasil, de administração totalmente pública. O levantamento de dados clínicos e sociodemográficos dos pacientes foi realizado a partir dos prontuários dos pacientes cadastrados no Polo I por meio de um instrumento de coleta de dados construído pelo pesquisador.

A coleta dos dados aconteceu no período de setembro a novembro de 2018. Os prontuários de pacientes com estomias que possuíam cadastro no Polo I foram incluídos, os de pacientes menos de 18 anos e os que já haviam realizado a reconstrução da estomia foram excluídos. Sendo assim, foram encontrados 270 prontuários, excluídos 13 por serem menor de 18 anos e dois por já terem realizado a reconstrução estomal, totalizando assim 255 prontuários selecionados.

Os dados coletados foram organizados e sumetidos a uma análise estatística descritiva, calculando-se valores percentuais e medidas de tendências centrais e de dispersão, utilizando-se o software Microsoft Excel 2010. Para a realização das análises estatísticas (teste qui-quadrado e teste exato de Fisher), o software GraphPad Prism 5.0 (GraphPad Software, Inc., USA) foi utilizado.

O estudo respeitou as exigências formais contidas nas normas nacionais e internacionais regulamentadoras de pesquisas envolvendo seres humanos e foi aprovado pelo Comitê de Ética e Pesquisa da Universidade Veiga de Almeida do Estado do Rio de Janeiro, com Parecer consubstanciado de no 2.907.189/2018. 


\section{Resultados}

A análise das características demográficas da população estudada apontou um maior número de atendimentos de pacientes em sua maioria do sexo masculino, sendo $138(54,1 \%)$ pessoas, $144(56,5 \%)$ com mais de 60 anos de idade e em média 71,1 anos de idade, 128 (50,2\%) brancos, 119 (46,7\%) com companheiro, $151(59,2 \%)$ com até o ensino fundamental, 88 (34,5\%) aposentados e 58 (22,7\%) sendo assegurado social, como demonstrado na Tabela 1.

Tabela 1 - Distribuição dos dados sociodemográficos das pessoas estomizadas. Cabo Frio, RJ, Brasil, 2018. $(\mathrm{n}=255)$

\begin{tabular}{lc}
\hline Características demográficas & $\mathbf{n}(\%)$ \\
\hline Gênero & \\
Masculino & $138(54,1)$ \\
Feminino & $117(45,9)$ \\
Faixa etária em anos (média de idade) & \\
$18-40(29,2 \pm 6,51)$ & $28(10,9)$ \\
$41-59(52,4 \pm 4,9)$ & $79(30,9)$ \\
60 ou mais $(71,1 \pm 8,0)$ & $144(56,5)$ \\
Não informado & $4(1,7)$ \\
Etnia & \\
Branco & $128(50,2)$ \\
Preto & $21(8,2)$ \\
Pardo & $98(38,4)$ \\
Não declarado & $8(3,1$ \\
Estado civil & \\
Sem companheiro & $69(27)$ \\
Com companheiro & $119(46,7)$ \\
Não declarado & $67(26,3)$ \\
Escolaridade & \\
Até ensino fundamental & $151(59,2)$ \\
Ensino Médio & $62(24,3)$ \\
Ensino Superior & $17(6,7)$ \\
Não informado & $25(9,8)$ \\
Profissão & \\
Desempregado & $47(18,4)$ \\
Aposentado & $88(34,5)$ \\
Assegurado social & $58(22,7)$ \\
Assalariado & $36(14,1)$ \\
Estudante & $2(0,8)$ \\
Não declarado & $24(9,4)$ \\
Total & $255(100,0)$ \\
\hline &
\end{tabular}

As análises dos dados clínicos dos estomas indicaram uma prevalência de pacientes com colostomia, $182(71,4 \%)$, e $177(69,4 \%)$ tendo a neoplasia como principal indicação para realização do estoma. Alguns problemas no processo do cuidar da pessoa com estomia podem impactar de forma negativa na vida do paciente, como o surgimento de complicações associadas às estomias. No presente estudo, as principais complicações encontradas foram a dermatite, ocorrendo em 44 pessoas $(17,3 \%)$, seguida pela presença de granuloma $(8,6 \%)$, prolapso $(8,2 \%)$ e hérnia $(7,1 \%)$. As demais complicações afetaram um percentual entre 4,3\% e 4,9\% dos indivíduos (Tabela 2).

As análises estatísticas (teste qui-quadrado e teste exato de Fisher) não apontaram nenhuma correlação entre a presença de estoma e o sexo $(p=0,165)$, idade $(\mathrm{p}=0,365)$ e escolaridade $(\mathrm{p}=0,663)$ dos pacientes. Observou-se uma correlação entre a presença de complicações e o sexo dos pacientes $(\mathrm{p}=0,037)$, porém não foram encontradas outras correlações entre as complicações e características sociodemográficas.

Tabela 2 - Caracterização das estomias. Cabo Frio, RJ, Brasil, 2018. $(n=255)$

\begin{tabular}{lc}
\hline Estoma & $\mathbf{n}(\%)$ \\
\hline Tipos & \\
Colostomia & $182(71,4)$ \\
Urestomia & $17(6,7)$ \\
Ileestomia & $27(10,6)$ \\
Transversestomia & $18(7,1)$ \\
Outros & $2(0,8)$ \\
Não informado & $9(3,5)$ \\
Indicação & \\
Perfuração & $19(7,5)$ \\
Neoplasia & $177(69,4)$ \\
Obstrução intestinal & $21(8,2)$ \\
Traumas & $6(2,4)$ \\
Outros & $22(8,6)$ \\
Não informado & $10(3,9)$ \\
Complicação & \\
Dermatite & $44(17,3)$ \\
Prolapso & $21(8,2)$ \\
Hérnia & $18(7,1)$ \\
Retração & $12(4,7)$ \\
Granuloma & $22(8,6)$ \\
Sangramento & $11(4,3)$ \\
Hiperemia & $15(5,9)$ \\
Outros & $13(5,1)$ \\
Não informado & $99(38,8)$ \\
Permanência & \\
Temporário & $86(33,7)$ \\
Definitivo & $77(30,2)$ \\
Indefinido & $58(22,7)$ \\
Outros & $34(13,3)$ \\
\hline &
\end{tabular}




\section{Discussão}

Os dados apontados pelo estudo são de representatividade local, o que não permite extrapolação para outras jurisdições. A coleta de dados foi realizada através de prontuários, configurando-se como limitação o preenchimento incompleto das informações sociais, dificultando a coleta e comprometendo a discussão de algumas variáveis previstas pela pesquisa. Fator este que justifica a necessidade de estudos epidemiológicos que visem levantar o perfil de saúde de uma determinada população a fim de dimensionar a problemática que os atinge, gerando assim dados relevantes para tomada de decisão por parte da equipe de saúde, em destaque o enfermeiro, que possui grande protagonismo no que diz respeito aos cuidados à pessoa estomizada.

Os dados coletados apontam para um número maior de pacientes do sexo masculino, o que se apresenta de forma similar em estudos anteriores, fato este que pode possuir relação com aumento da violência urbana, uso de drogas lícitas ou ilícitas, menor procura e utilização dos serviços de saúde e serviços de prevenção e procura tardia do atendimento médico, situações mais comumente observadas em homens $^{(4-5)}$. Embora evidências científicas apontem para um predomínio de idosos portadores de doenças que levem à constituição de estomias ${ }^{(6)}$, a necessidade de atenção à prevenção de doenças e promoção da saúde destinada aos indivíduos das demais faixas etárias permanece.

A predominância de pacientes brancos encontrada neste estudo se contrapõe aos dados disponíveis na literatura que apontam para uma maior quantidade de negros ou pardos portadores de estomias ${ }^{(7)}$. Uma possível hipótese para tal contradição pode ser o fato da etnia ser autodeclarada, a partir de como o paciente se percebe. Uma vez que nosso país apresenta uma elevada miscigenação e a classificação de cor e raça é, quase exclusivamente, declarada pelo que se entende da cor da pele. É possível que a percepção individual do paciente de sua tonalidade de pele seja responsável por essa contradição nos dados frente à literatura disponível.

De maneira similar a estudos anteriores, a maior parte dos pacientes possui baixo nível de escolaridade que, em geral, resulta em maior dificuldade de compreender os problemas de saúde e processos de prevenção em saúde. Além disso, a manutenção de hábitos prejudiciais como etilismo, tabagismo, sedentarismo e maus hábitos alimentares é maior entre tal perfil demográfico, fatores intimamente ligados às neoplasias malignas como o câncer colorretal, principal responsável pela realização de colostomias ${ }^{(2,8)}$. Os resultados obtidos no presente estudo reforçam, portanto, dados disponíveis na literatura que indicam a escolaridade reduzida e a idade avançada como fatores sociodemográficos ligados à presença de esto$\operatorname{mias}^{(2-3)}$.

A análise do perfil profissional revelou pacientes em sua maioria aposentados ou assegurados sociais por deficiência, similar ao perfil previamente descrito em outros estudos ${ }^{(9-10)}$. É possível que o perfil profissional encontrado ocorra devido à idade majoritária dos pacientes ser de mais de 60 anos. Porém, vale ressaltar que, pacientes estomizados podem receber auxílio do governo pelo período da doença ou até permanentemente, com base no Decreto $\mathrm{n}^{\circ} 5.296$, de 2 de dezembro de 2004. Tal legislação visa assegurar tal direito às pessoas portadoras de deficiências, sendo esta entendida pela lei como qualquer alteração parcial ou completa de um ou mais seguimentos do corpo humano que implique em comprometimento da função orgânica, como paraplegia, paraparesia, monoplegia, monoparesia, tetraplegia, tetraparesia, triplegia, triparesia, hemiplegia, hemiparesia e ostomia, dentre outros ${ }^{(11)}$.

Além disso, o perfil profissional encontrado como sendo maioria aposentados ou assegurado social pode estar associado ao desafio que estes indivíduos encontram para conseguir emprego ou retonar às suas atividades laborais, visto que tal quadro possui uma relação direta com a autoimagem e a readaptação ao estilo de vida. Neste mesmo contexto, um estudo 
corrobora com dados desta pesquisa, o qual afirma que a estomia torna-se um fator incapacitante para desenvolvimento da atividade laboral e tem um relação direta com a queda na qualidade de vida ${ }^{(9)}$. Outro estudo desenvolvido na cidade de São Paulo aponta que a maioria da pessoas com estomia não retornam a sua atividade laboral, gerando um grande conflito no processo de reabilitação dessa pessoa ${ }^{(12)}$. Neste cenário, é de suma importância que o enfermeiro possa implementar medidas socias para o perfil de pacientes atendidos, visto que a renda e sua atividade laboral podem possuir uma estreita relação com a adesão do paciente aos cuidados relacionados à estomia.

Portanto, os profissionais de saúde, em especial o enfermeiro, devem considerar esta realidade ao prestar atendimento e cuidados ao paciente estomizado, visando o enfrentamento e adaptação do paciente a essa nova realidade, inclusive no que diz respeito à atividade profissional do indivíduo.

Os tipos de estomias encontradas também corroboram dados previamente publicados, indicando que estomas digestivos de eliminação, também conhecido como colostomia, é o procedimento mais comum do trato digestório, causado principalmente por obstruções, traumas, doenças inflamatórias e neoplasias $^{(12)}$. O câncer colorretal é o principal causador da necessidade de um estoma, sendo, excetuando-se o câncer de pele não melanoma, o segundo e terceiro tipo de câncer mais incidente em mulheres e homens, respectivamente ${ }^{(13)}$. É importante salientar que a incidência do câncer vem aumentando ao longo dos anos, que possivelmente está associada a uma maior expectativa de vida e hábitos e estilo de vida da população induzem mudanças importantes em seu corpo $^{(14)}$. Tal aumento pode impactar também no número de pacientes estomizados, o que reforça ainda mais a necessidade do enfermeiro conhecer o perfil e estar preparado para lidar com estes pacientes, instituindo medidas de promoção da saúde e prevenção de agravos da doença.

A dermatite irritativa é um dos problemas mais frequentes nos estomizados, e em geral corre devido ao uso inadequado dos equipamentos, corte inadequado do orifício da bolsa ou má instalação desta, fazendo com que o fluído intestinal permaneça em contato com a pele ao redor do estoma, irritando- $\mathrm{a}^{(2)}$. Assim como demonstrado em estudos anteriores ${ }^{(2,15)}$, a dermatite irritativa foi a complicação mais incidente dentre os pacientes analisados no presente estudo. A prevenção do surgimento da dermatite e lesões na pele deve ser a meta fundamental, sendo imprescindível que o paciente seja corretamente orientado quanto ao autocuidado ${ }^{(2)}$.

Nesse sentido, a equipe interdisciplinar exerce papel fundamental, sendo o profissional presente desde o diagnóstico e realização do estoma ainda no ambiente hospitalar, durante o período de hospitalização e nas unidades especializadas, postos de saúde e equipes de saúde da família acompanhando o pós-operatório tardio dos indivíduos estomizados ${ }^{(16)}$. Vale ressaltar que, uma correlação entre o sexo dos pacientes e a presença de complicações foi encontrada durante o presente estudo, indicando que o sexo do paciente pode ter impactado no surgimento de complicações das estomias. As evidências disponíveis possuem pouca concordância na correlação do sexo com fatores de risco associados a complicações ${ }^{(17)}$. Este fato se torna um alvo em potencial para implementações de medidas de enfermagem que possam preencher esta lacuna no cuidar.

Existem diversos fatores associados ao desenvolvimento de complicações vinculadas com a estomia, a maioria não modificais, porém, ainda existem fatores assistenciais relevantes, principalmente sobre a tríade do cuidado interal enfermagem (paciente, profissional e família) na redução da incidência das complicações e detecção precoce, se tornando potencial para melhoria da qualidade de vida da pessoa com estomia $^{(17)}$.

Com relação à permanência dos estomas, em sua maioria foram encontrados estomas temporários, o que corrobora dado já descrito na literatura(7). Po- 
rém, contrapondo com outro achado, em que grande parte dos estomas, principalmente intestinais, eram de caráter definitivo ${ }^{(9)}$, sustentando a necessidade de pautar os cuidados de saúde no perfil da população assistida.

A confecção dos estomas e o seu tempo de permanência é um grande desafio para o paciente, no que tange ao processo de aceitação da condição atual de saúde e as incertezas do futuro. A média do tempo de permanência de estomias temporárias é de cerca de cinco anos ${ }^{(12)}$, e as pessoas que se submetem à estomização, nos primeiros meses, tendem a apresentar um abatimento e até mesmo revolta com a presença do estoma, o que as leva a questionar o sentido da cirurgia $^{(16)}$. Nesta fase, o profissional de saúde se destaca como um agente de transformação e importante facilitador para a aceitação do estoma, seja ele temporário ou permanente.

\section{Conclusão}

Os dados mostram como perfil dos indivíduos da população estomizada um paciente predominantemente idoso, do sexo masculino, branco, com companheiro, aposentado e de baixa escolaridade, com a presença de estomas intestinais devido à presença de neoplasia, sendo tal estoma de permanência temporária e tendo como principal complicação a dermatite irritativa decorrente do contato constante da pele com o fluído intestinal. Logo, existiu uma relação entre o gênero e as complicações apresentadas.

\section{Colaborações}

Cerqueira LCN, Cacholi SAB e Nascimento VS colaboraram na concepção e projeto, análise e interpretação dos dados. Todas as autoras contribuíram para a redação do artigo ou revisão crítica relevante do conteúdo intelectual. Koeppe GBO, Oliveira PP e Torres VCP contribuíram na aprovação final da versão a ser publicada.

\section{Referências}

1. World Health Organization. Saving lives, spending less. A strategic response to noncommunicable diseases [Internet]. 2018 [citado 2019 nov 16]. Disponível em: https://apps.who.int/iris/bitstream/handle/10665/272534/WHO-NMH-NVI-18.8-eng.pdf?ua=1

2. Miranda SM, Luz HBA, Sonobe HM, Andrade EMLR, Moura ECC. Caracterização sociodemográfica e clínica de pessoas com estomia em Teresina. Estima. 2016; 14(1):29-35. doi: http://dx.doi. org/10.5327/Z1806-3144201600010005

3. Stricher L, Hocevar B, Asburn J. Fecal and urinary stoma construction. In: Carmel JE, Colwell JC, Goldberg MT, eds. Wound ostomy and continence nurses society core curriculum: ostomy management. Philadelphia: Lippincott Williams and Wilkins; 2016.

4. Barbosa MR, Simon BS, Tier CG, Garcia RP, Siniak DS, Rodrigues SO. Profile of people with stomas from a municipal health service in Southern of Brazil. ESTIMA Braz J Enterostomal Ther. 2018; 16:e1318. doi: http://dx.doi.org/10.30886/ estima.v16.465_PT

5. Secretaria do Estado de Saúde (RJ). Retratos municipais - baixada litorânea [Internet]. [citado 2019 jul. 20]. Disponível em: http://sistemas. saude.rj.gov.br/tabnet/retratos/BAIXADA_ LITORANEA/BLIT.html

6. Moraes KL, Brasil VV, Oliveira GF, Cordeiro JABL, Silva AMTC, Boaventura RP, et al. Functional health literacy and knowledge of renal patients on pre-dialytic treatment. Rev Bras Enferm. 2017; 70(1):147-53. doi: http://dx.doi. org/10.1590/0034-7167-2015-0169

7. Ecco L, Dantas FG, Melo MDM, Freitas LS, Medeiros LP, Costa IKF. Perfil de pacientes colostomizados na Associação dos Ostomizados do Rio Grande do Norte. ESTIMA Braz J Enterostomal Ther. 2018; 16:e0518. doi: http://dx.doi.org/10.30886/ estima.v16.351_PT

8. Nahas SC, Nahas CSR, Bustamante-Lopez LA, Pinto RA, Marques CFS, Campos FG, et al. Prognostic factors of surgically-treated patients with cancer of the right colon: a ten years' experience of a 
single universitary institution. Arq Bras Cir Dig. 2015; 28(1):3-7. doi: http://dx.doi.org/10.1590/ S0102-67202015000100002

9. Miranda LSG, Carvalho AAS, Paz EPA. Quality of life ofostomized person:relationshipwith the careprovided in stomatherapy nursing consultation. Esc Anna Nery. 2018; 22(4):e20180075. doi: http:// dx.doi.org/10.1590/2177-9465-EAN-2018-0075

10. Marques GS, Nascimento DC, Rodrigues FR, Lima CMF, Jesus DF. A vivência de pessoas com estomia intestinal no grupo de apoio em um Hospital Universitário. HUPE. 2016; 15(2):113-21. doi: http://dx.doi.org/10.12957/rhupe.2016.28235

11. Presidência da República (BR). Decreto n 5.296 , de 2 de dezembro de 2004: regulamenta as Leis no 10.048 , de 8 de novembro de 2000 , que dá prioridade de atendimento às pessoas que especifica, e 10.098, de 19 de dezembro de 2000, que estabelece normas gerais e critérios básicos para a promoção da acessibilidade das pessoas portadoras de deficiência ou com mobilidade reduzida, e dá outras providências. Brasília: Casa Civil; 2004.

12. Aguiar JC, Pereira APS, Galisteu KJ, Lourenção LG, Pinto MH. Clinical and sociodemographic aspects of people with a temporary intestinal stoma. Rev Min Enferm. 2017; 21:e-1013. doi: http://dx.doi. org/10.5935/1415-2762.20170023
13. Ministério da Saúde (BR). Instituto Nacional de Câncer José Alencar Gomes da Silva. Coordenação de Prevenção e Vigilância. Estimativa 2018: incidência de câncer no Brasil. Rio de Janeiro: INCA; 2017.

14. Panis C, Kawasaki AC, Pascotto CR, Justina EY, Vicentini GE, Lucio LC, et al. Critical review of cancer mortality using hospital records and potential years of life lost. Einstein (São Paulo). 2018; 16(1):eA04018. doi: http://dx.doi.org/10.1590/ s1679-45082018ao4018

15. Santos VLCG, Cesaretti IUR. Assistência em estomaterapia - cuidando de pessoas com estomia. São Paulo: Atheneu; 2015.

16. Silva NM, Santos MA, Rosado SR, Galvão CM, Sonobe HM. Psychological aspects of patients with intestinal stoma: integrative review. Rev LatinoAm Enfermagem. 2017; 25:e2950. doi: http:// dx.doi.org/10.1590/1518-8345.2231.2950

17. Pinto IES, Queirós SMM, Queirós CDR, Silva CRR, Santos CSVB, Brito MAC. Risk factors associated with the development of elimination stoma and peristomal skin complications. Referência. 2017; IV(15):155-66. doi: https://doi.org/10.12707/ RIV17071

\section{(cc) BY}

Este é um artigo de acesso aberto distribuído sob os termos da Licença Creative Commons 